\title{
Incremental Cost-Effectiveness of 13-valent Pneumococcal Conjugate Vaccine for Adults Age 50 Years and Older in the United States
}

\author{
Charles Stoecker, $\mathrm{PhD}^{7}$, Lindsay Kim, MD, $\mathrm{MPH}^{2}$, Ryan Gierke, $\mathrm{MPH}^{2}$, and Tamara Pilishvili, $\mathrm{MPH}^{2}$ \\ 'Department of Global Health Management and Policy, Tulane University School of Public Health and Tropical Medicine, New Orleans, LA, USA; \\ ${ }^{2}$ National Center for Immunization and Respiratory Diseases, Centers for Disease Control and Prevention, Atlanta, GA, USA.
}

\begin{abstract}
BACKGROUND: Recently released results from a randomized controlled trial have shown that 13-valent pneumococcal conjugate vaccine (PCV13) is efficacious against vaccine-type nonbacteremic pneumonia in adults.

OBJECTIVE: We examined the incremental costeffectiveness of adding PCV13 to the Advisory Committee on Immunization Practices (ACIP) adult immunization schedule.
\end{abstract}

METHODS: We used a probabilistic model following cohorts of 50-, 60-, or 65-year-olds. We used separate vaccination coverage and disease incidence data for healthy and highrisk adults. Incremental cost-effectiveness ratios were determined for each potential vaccination strategy.

RESULTS: In the base case scenario, our model indicated that adding PCV13 at age 65 or replacing 23-valent pneumococcal polysaccharide vaccine (PPSV23) at age 65 with PCV13 provided more value for money than adding PCV13 at ages 50 or 60. After projections of six additional years of herd protection from the childhood immunization program were incorporated, we found adding PCV13 dominated replacing PPSV23. For a cohort of 65-yearolds in 2013, the cost of adding PCV13 at age 65 to the schedule was $\$ 62,065$ per quality-adjusted life year (QALY) gained, which rose to $\$ 272,621$ after 6 years of projected herd protection.

CONCLUSION: The addition of one dose of PCV13 for adults appears to have a cost-effectiveness ratio comparable to other vaccination interventions in the short run, though anticipated herd protection from the childhood immunization program may dramatically increase the cost per GALY after only a few years.

KEY WORDS: vaccinations; cost-effectiveness; pneumococcal disease. $\mathrm{J}$ Gen Intern Med 31(8):901-8

DOI: $10.1007 / \mathrm{s} 11606-016-3651-0$

(c) Society of General Internal Medicine 2016

\section{INTRODUCTION}

In 2013, pneumonia accounted for 53,282 deaths in the United States ${ }^{1}$ and the total medical cost of pneumonia in the fee-for-

Electronic supplementary material The online version of this article (doi:10.1007/s11606-016-3651-0) contains supplementary material, which is available to authorized users.

Received August 24, 2015

Revised December 30, 2015

Accepted February 18, 2016

Published online March 14, 2016 service Medicare population was estimated in excess of $\$ 7$ billion in $2010 .^{2}$ The introduction of 13 -valent pneumococcal conjugate vaccine (PCV13) to the recommended schedule for children significantly reduced the all-cause pneumonia hospitalizations for children burden, while it remained high among older adults. ${ }^{3}$

In 2014, the Advisory Committee on Immunization Practices (ACIP) voted to add a single dose of PCV13 to the existing recommendation of 23-valent pneumococcal polysaccharide vaccine (PPSV23) for all adults over the age of 65 years. ${ }^{4}$ Recent evidence has shown that PCV13 is efficacious against vaccine-type community acquired pneumonia, ${ }^{5}$ which remains prevalent among older adults despite recently observed declines due to herd protection provided by use of PCV7 and PCV13 among children. ${ }^{3,6}$ While previous studies have examined the cost-effectiveness of vaccinating old adults with PCV13, ${ }^{7}$ this study fills a gap in the literature by incorporating clinical trial evidence of PCV13 effectiveness against community acquired pneumonia. We aimed to evaluate the marginal cost-effectiveness of the new recommendation as well as three alternate strategies to add PCV13 to the immunization schedule for adults. We evaluated adding PCV13 at ages 50, 60, or 65 years to the existing PPSV23 recommendation, as well as a strategy that replaces PPSV23 at age 65 with PCV13 at age 65.

\section{METHODS}

\section{Model}

We developed a probabilistic model to estimate the costeffectiveness of altering the pneumococcal vaccination schedule for adults to include PCV13 for hypothetical cohorts of adults. We used Monte Carlo simulation in spreadsheet-based software $^{8}$ to estimate the impact on program costs, medical costs, non-medical costs, and disease burden when adding a dose of PCV13 to the previously existing PPSV23 recommendation or replacing PPSV23 with PCV13 at age 65 years.

We tracked new disease incidence, including cases and deaths due to invasive pneumococcal disease (IPD) and nonbacteremic pneumococcal pneumonia (NBP), through life expectancy or until age 100 . We modeled the effects of several 
recommended schedules: (1) a dose of PCV13 followed by a dose of PPSV23 at age 65 in a cohort of 65-year-olds, (2) a dose of PCV13 at age 50 followed by a dose of PPSV23 at age 65 in a cohort of 50-year-olds, (3) a dose of PCV13 at age 60 followed by a dose of PPSV23 at age 65 in a cohort of 60 -yearolds, and (4) a dose of PCV13 at age 65 in a cohort of 65-yearolds. Each of these hypothetical strategies preserved the riskbased recommendations for adults 19-64 years of age. ${ }^{9} \mathrm{We}$ calculated the incremental cost-effectiveness of each of these strategies compared to the current recommendation of a dose of PPSV23 at diagnosis of high-risk condition for ages 50-64, followed by a dose of PPSV23 at age 65 (or 5 years later). Our analysis was conducted from a societal perspective, which included the totality of societal costs and benefits. The general model structure is shown in Fig. 1.

\section{Study Population}

We focused on three cohorts of adults the size of the United States population in 2013: 4,494,482 50-year-olds, 3,862,796 60-year-olds, and 3,391,067 65-year-olds. From these groups, we subtracted the immunocompromised population as individuals developed immunocompromising conditions, since this group was recommended to receive PCV13 in 2012 and the cost effectiveness of that recommendation has already been evaluated in previous work. ${ }^{10}$ We stratified the remaining immunocompetent adults by risk status. We classified individuals with four chronic conditions as high-risk: diabetes, chronic heart disease, chronic lung disease, and chronic liver disease. Our model followed individuals by single year of age and through life expectancy or age 100, whichever came first.

\section{Disease Parameters}

For IPD, we classified serotypes as (1) those included in PCV13 (1, 3, 4, 5, 6A, 6B, 7F, 9V, 14, 18C, 19A, 19F, 23F), and (2) those included in PPSV23 $(1,2,3,4,5,6 \mathrm{~B}, 7 \mathrm{~F}, 8,9 \mathrm{~N}$, $9 \mathrm{~V}, 10 \mathrm{~A}, 11 \mathrm{~A}, 12 \mathrm{~F}, 14,15 \mathrm{~B}, 17 \mathrm{~F}, 18 \mathrm{C}, 19 \mathrm{~F}, 19 \mathrm{~A}, 20,22 \mathrm{~F}, 23 \mathrm{~F}$ and $33 \mathrm{~F}$ ). The number of IPD cases by these serotype classifications among the healthy and high-risk populations was obtained from the Centers for Disease Control and Prevention's Active Bacterial Core surveillance $(\mathrm{ABCs})^{11}$ in 2013. Rates of NBP were taken from previously published reports. ${ }^{3,}$

${ }^{12}$ Age and risk-specific disease rates are displayed in Table 1.

We modified the resulting IPD and NBP rates to account for projected changes in IPD incidence in the adult population due to the introduction of PCV13 for children in $2010 .{ }^{19}$ The rates used in our calculations are displayed in Table 1. As 2013 was the most recent year with available data, we predicted forward from 2013. To predict forward, we first calculated the declines of PCV7-type IPD among adults between 2003 and 2009. We assumed the resulting declines of $78.6 \%$ for 50-64-year-olds and $86.6 \%$ for $\geq 65$-year-olds would be observed for the additional serotypes in PCV13 between 2013 and 2019. We estimated that serotypes in PPSV23, but not in PCV13, increased by $77.9 \%$ for $50-64$-year-olds and $17 \%$ for $\geq 65$ - year-olds between 2003 and 2009. We applied this increase to the 2013 IPD incidence for serotypes in PPSV23 but not in PCV13 starting in 2019, to account for serotype replacement disease. We scaled IPD incidence for each serotype grouping (types in PCV13 not in PCV7, serotypes in PPSV23 not in PCV13, and serotypes in neither vaccine) linearly between years 0 and 6, using reported incidence from 2013 as year 0 to the full herd protection calculated in year 6 to account for the fact that our serotype changes were measured over a 6-year period. We did not assume any further changes in serotype distribution due to herd protection from the childhood program after year 6 .

\section{Vaccine Effectiveness and Coverage}

Estimates of PCV13 effectiveness against IPD and NBP were based on results from the CAPiTA trial. ${ }^{5}$ This was a randomized, double-blind, placebo-controlled trial designed to evaluate the effectiveness of PCV13 against IPD and NBP. We based the effectiveness of PPSV23 against IPD on results from a systematic review of 18 randomly controlled trials and seven observational studies, ${ }^{14}$ and NBP on results from a systematic review of 19 trials \{Huss, $2009 \# 105$ \}. Effectiveness by age and risk group is detailed in Table 1.

We assumed PCV13 exhibited no declines in effectiveness between the ages of 50 and 65 . We made this assumption since we lacked vaccine effectiveness data at age 50. This assumption tilts recommendations slightly in favor of vaccination at age 50 . After age 65 , effectiveness waned by $10 \%$ every 5 years, with this waning distributed linearly within each 5 year increment. We also investigated a scenario where PCV13 showed no waning for the first 5 years after age 65 , followed by a linear decline to zero effectiveness over the next 15 years. We assumed PPSV 23 waned linearly to $50 \%$ of initial effectiveness over the first 5 years, linearly to $30 \%$ of initial effectiveness over the next 5 years, and linearly to $0 \%$ effectiveness over the next 5 years. ${ }^{20}$ We tracked vaccine waning separately for each vaccine and always incorporated the most generous protection for each serotype group.

Coverage rates for the population over age $65(59.9 \%)$ and high-risk 50-64-year-olds (20\%) were based on the 2012 National Health Interview Survey. ${ }^{16}$ As healthy 50-64-yearolds are not currently recommended to receive pneumococcal vaccination, we constructed two plausible bounds for the coverage rate. For a lower bound estimate of coverage in healthy 50-64-year-olds, we used the observed coverage rate in high-risk 50-64-year-olds. For the upper bound, we multiplied the observed coverage rate for the population over age 65 by the ratio of the percent of 45-64-year-olds that had seen a doctor in the last year ( $84 \%$ ), to the percent of 65-75-yearolds that had seen a doctor in the last year $(92.5 \%)$. The percent of the population with doctor visits in the last year was also taken from the 2012 National Health Interview Survey. ${ }^{17}$ To obtain a base case coverage rate in healthy 50 64-year-olds, we averaged the upper and lower bounds. 


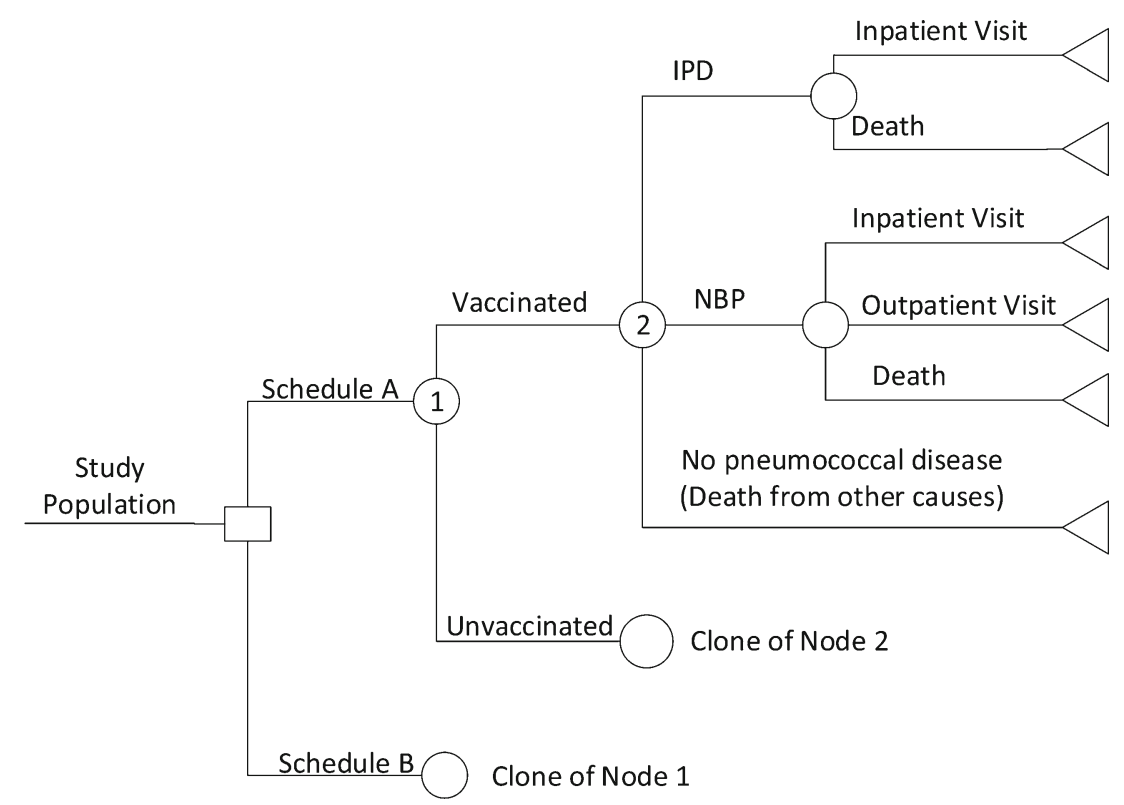

Figure 1. Model structure. Notes: Figure shows pneumococcal related outcomes in the cost-effectiveness model. Abbreviations: IPD invasive pneumococcal disease, NBP non-bacteremic pneumococcal pneumonia.

\section{Health Utility Indices}

To assess the impact of vaccination across a variety of outcomes and populations, we used quality-adjusted life year (QALY) decrements. Decrements could range from 0 for a person in perfect health to 1 for death. We used two sources for QALY decrements for pneumococcal diseases. Our base case uses QALY decrements of 0.006 for inpatient NBP, and 0.004 for outpatient $\mathrm{NBP}^{21}$ We arrived at a QALY decrement of 0.009 for IPD by weighting the QALY decrements for bacteremia and meningitis by their respective case counts in 2013 ABCs surveillance data. These QALY decrements correspond to 3.2 days of healthy life lost for IPD, 2.2 days of healthy life lost for inpatient NBP, and 1.5 days of healthy life lost for outpatient NBP. These QALY decrements can be put into context by assuming that inpatient pneumococcal related illness results in quality of life losses of $15 \%$ and outpatient pneumococcal illness results in quality of life losses of $10 \%{ }^{22}$ These imply average illness durations of 27 days for inpatient disease and 18 days for outpatient disease for otherwise healthy people .

We also investigated an alternative scenario with much larger disease QALY decrements of 0.075 for IPD and 0.075 for inpatient NBP. ${ }^{23}$ We created a QALY decrement of 0.050 for outpatient NBP in this second QALY scenario by modifying the outpatient QALY decrement in the base case by the ratio of the inpatient NBP QALY decrement in the second scenario to the inpatient NBP QALY decrement in the base case. The implied illness durations for this second set of QALY decrements was 181 days across both inpatient and outpatient diseases.

To determine the QALY decrement for each episode of illness, we multiplied the baseline QALY for a given age category and risk status by utility decrements. Baseline age and risk status specific QALY values have been previously published. $^{23}$

\section{Medical and Non-medical Costs}

Inpatient and outpatient costs from clinic visits were obtained from paid health insurance claims from the Truven Health Analytics MarketScan database 2010. ${ }^{18}$ We calculated costs for these patients when they had IPD (International Classification of Diseases, Ninth Revision, Clinical Modification codes 320.1, 038.2), or all-cause pneumonia (codes 481, $3.22,11.6,20.3,20.4,20.5,55.1,73.0,112.4,115.05$, $115.15,115.95,130.4,136.3,480,482,483,484,485,486$, and 487.0). Costs were averaged across 50-64-year-olds and $\geq$ 65-year-olds separately.

For vaccine cost, we applied government contract vaccine prices published annually of $\$ 85.189$ in $2013 .{ }^{24}$ For travel and vaccine administration cost, we used estimates from previously published sources, ${ }^{25}$ which after adjustment to $2013 \$$ were $\$ 23$ and \$17, respectively. All outcomes were discounted by $3 \%$ and adjusted to $2013 \$$ using the Consumer Price Index for all items. ${ }^{26}$ We did not calculate the effects of vaccine wastage or adverse events. In pre-licensure studies, serious adverse events resulting from receipt of PCV13 were uncommon, ${ }^{27}$ and both vaccines showed similar rates of adverse events. ${ }^{28,29}$

\section{Sensitivity Analyses}

We conducted multivariate sensitivity analysis around our inputs according to the ranges indicated in Table 1. For cost parameters, we used the ranges indicated in Table 1 as the 5th and 95 th percentiles for a log-normal distribution. The 5 th and 95th percentiles of the sample mean were calculated by bootstrapping the mean MarketScan data over 1000 repetitions with a sample size of 100 . To reflect the uncertainty in 
Table 1. Age (in Years) and Risk-Specific Disease Rate, Vaccine Effectiveness, Coverage Rate, and Cost Inputs for Prediction Model

\begin{tabular}{|c|c|c|c|c|c|c|c|c|}
\hline \multicolumn{9}{|l|}{ Disease } \\
\hline & \multicolumn{2}{|c|}{ Age 50-64, Healthy } & \multicolumn{2}{|c|}{$\begin{array}{l}\text { Age } 50-64 \text {, High-risk } \\
\text { base range }\end{array}$} & \multicolumn{2}{|c|}{ Age $\geq 65$, Healthy } & \multicolumn{2}{|c|}{ Age $\geq 65$, High-risk } \\
\hline $\operatorname{IPD}$ rate $(\text { ner } 100000)^{\mathbf{a}}$ & 8.62 & $6.5-8.94$ & 33.71 & $32.33-34.6$ & 15.06 & $13.25-16.04$ & 47.63 & $\begin{array}{l}\text { range } \\
47.63-62.82\end{array}$ \\
\hline$\%$ IPD cases resulting in fatality & \multicolumn{2}{|l|}{$\begin{array}{l}8.62 \\
6.74\end{array}$} & \multicolumn{2}{|l|}{11.62} & \multicolumn{2}{|l|}{11.85} & \multicolumn{2}{|l|}{15.05} \\
\hline \% PCV13 serotype IPD & \multicolumn{2}{|l|}{26.75} & \multicolumn{2}{|l|}{24.8} & \multicolumn{2}{|l|}{24.7} & \multicolumn{2}{|l|}{21.32} \\
\hline \% PPSV23 serotype IPD & \multirow{2}{*}{\multicolumn{2}{|c|}{$\begin{array}{l}74.52 \\
48.09\end{array}$}} & \multirow{2}{*}{\multicolumn{2}{|c|}{$\begin{array}{l}69.19 \\
4439\end{array}$}} & \multicolumn{2}{|l|}{62.35} & \multicolumn{2}{|l|}{59.69} \\
\hline \% PPSV23 serotype IPD (but not in & 48.09 & & & & \multirow{2}{*}{\multicolumn{2}{|c|}{38.06}} & \multicolumn{2}{|l|}{38.37} \\
\hline PCV13) & & \multicolumn{2}{|l|}{44.39} & & & \multirow{2}{*}{\multicolumn{2}{|c|}{40.31}} \\
\hline \% Nonvaccine Serotype IPD & & & \multicolumn{2}{|l|}{30.81} & \multicolumn{2}{|l|}{37.25} & & \\
\hline Inpatient NBP rate $(\text { per } 100,000)^{b}$ & 258.2 & $258.2-267.7$ & 258.2 & $258.2-267.7$ & 1375.2 & $\begin{array}{l}1375.2- \\
1438.4\end{array}$ & 1375.2 & $\begin{array}{l}1375.2- \\
1438.4\end{array}$ \\
\hline$\%$ NBP cases resulting in fatality ${ }^{c}$ & 3.2 & $2-4$ & 3.2 & $2-4$ & 6.7 & $5-7$ & 6.7 & $5-7$ \\
\hline Outpatient NBP rate $(\text { per } 100,000)^{\mathrm{d}}$ & 600 & & 600 & & 2010 & & 2010 & \\
\hline$\%$ NBP due to PCV13 types ${ }^{\mathrm{e}}$ & 10 & $9-14$ & 10 & $9-14$ & 10 & $9-14$ & 10 & $9-14$ \\
\hline Serotype changes between 2003 and $2009^{\mathrm{a}}$ & & & & & & & & \\
\hline $\begin{array}{l}\text { (cases per } 100,000) \\
\text { Year }\end{array}$ & Age 50 & 64, Healthy & Age 5( & 64, High-risk & Age $\geq$ & 5, Healthy & Age $\geq$ & , High-risk \\
\hline Year & 2003 & 2009 & 2003 & 2009 & 2003 & 2009 & 2003 & 2009 \\
\hline PCV7 types & 6.26 & 1.34 & 6.26 & 1.34 & 13.62 & 1.82 & 13.62 & 1.82 \\
\hline PPSV23 types not in PCV7 & 8.35 & 14.85 & 8.35 & 14.85 & 20.83 & 24.38 & 20.83 & 24.38 \\
\hline Types in neither PCV7 nor PPSV23 & 3.61 & 5.01 & 3.61 & 5.01 & 7.67 & 12.62 & 7.67 & 12.62 \\
\hline & $\begin{array}{l}\text { Age } 50 \\
\text { base }\end{array}$ & $\begin{array}{l}64 \text {, Healthy } \\
\text { range }\end{array}$ & $\begin{array}{l}\text { Age } 5( \\
\text { base }\end{array}$ & $\begin{array}{l}\text { 64, High-risk } \\
\text { range }\end{array}$ & $\begin{array}{l}\text { Age } \geq \\
\text { base }\end{array}$ & $\begin{array}{l}5 \text {, Healthy } \\
\text { range }\end{array}$ & $\begin{array}{l}\text { Age } \geq \\
\text { base }\end{array}$ & $\begin{array}{l}\text {, High-risk } \\
\text { range }\end{array}$ \\
\hline PCV vs. VT IPD & 75.00 & $41.43-90.78$ & 75.00 & $41.43-90.78$ & 75.00 & $41.43-90.78$ & 75.00 & $41.43-90.78$ \\
\hline PCV vs. VT NBP & 45.00 & $14.21-65.31$ & 45.00 & $14.21-65.31$ & 45.00 & $14.21-65.31$ & 45.00 & $14.21-65.31$ \\
\hline PPSV vs. VT IPD ${ }^{\mathrm{f}}$ & 74.00 & $64-85$ & 74.00 & $48-85$ & 74.00 & $64-85$ & 74.00 & $64-85$ \\
\hline PPSV vs. VT NBP ${ }^{g}$ & 0 & $0-50$ & 0 & $0-50$ & 0 & $0-50$ & 0 & $0-50$ \\
\hline 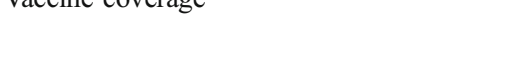 & Age 50 & 64, Healthy & Age 5 & 64, High-risk & Age $\geq$ & 5 , Healthy & Age $\geq$ & , High-risk \\
\hline Coverage rate $(\%)^{\mathrm{h}}$ & $\begin{array}{l}\text { base } \\
27.2\end{array}$ & $20-54.4$ & & $\begin{array}{l}\text { range } \\
18.9-21.1\end{array}$ & $\begin{array}{l}\text { base } \\
59.9\end{array}$ & $\begin{array}{l}\text { range } \\
58.4-61.4\end{array}$ & $\begin{array}{l}\text { base } \\
59.9\end{array}$ & $\begin{array}{l}\text { range } \\
58.4-61.4\end{array}$ \\
\hline Cost per case, $2010 \$^{j}$ & & & & & & & & \\
\hline & $\begin{array}{l}\text { Age } 50 \\
\text { base }\end{array}$ & $\begin{array}{l}64 \text {, Healthy } \\
\text { range }\end{array}$ & $\begin{array}{l}\text { Age } 5( \\
\text { base }\end{array}$ & $\begin{array}{l}\text { 64, High-risk } \\
\text { range }\end{array}$ & $\begin{array}{l}\text { Age } \geq \\
\text { base }\end{array}$ & $\begin{array}{l}\text {, Healthy } \\
\text { range }\end{array}$ & $\begin{array}{l}\text { Age } \geq \\
\text { base }\end{array}$ & 5, High-risk \\
\hline IPD & 40,161 & $\begin{array}{l}31,950- \\
49,086\end{array}$ & 40,161 & $\begin{array}{l}31,950- \\
49,086\end{array}$ & 27,097 & $\begin{array}{l}21,981- \\
32,695\end{array}$ & 27,097 & $\begin{array}{l}21,981- \\
32,695\end{array}$ \\
\hline Inpatient NBP & 34,948 & $\begin{array}{l}25,468- \\
46,117\end{array}$ & 34,948 & $\begin{array}{l}25,468- \\
46,117\end{array}$ & 23,296 & $\begin{array}{l}16,916- \\
31.696\end{array}$ & 23,296 & $\begin{array}{l}16,916- \\
31.696\end{array}$ \\
\hline Outpatient NBP & 127 & $77-219$ & 127 & $77-219$ & 254 & $121-463$ & 254 & $121-463$ \\
\hline
\end{tabular}

Abbreviations: IPD invasive pneumococcal disease, PCV13 13-valent pneumococcal conjugate vaccine, PPSV23 23-valent pneumococcal polysaccharide vaccine, NBP non-bacteremic pneumococcal pneumonia, VT vaccine type

${ }^{a}$ Source: ABCs surveillance data 2013. ${ }^{11}$ Note inputs from ABCs are displayed here for reproducibility of final results. The number of digits displayed should not necessarily be construed as a measure of the accuracy of these outputs from ABCs surveillance data

${ }^{b}$ Source: Simonsen et al. $2014^{3}$

c Source: Huang et al. $2011^{13}$

${ }^{d}$ Source: Nelson et al. $2008^{12}$

e Source: CAPITA

${ }^{f}$ Source: Moberley et al. $2008^{14}$

${ }^{g}$ Source: Huss et al. $2009^{15}$

${ }^{h}$ Source: National Health Interview Survey 2012 ${ }^{16,17}$

${ }^{j}$ Source: MarketScan $2010^{18}$

QALY parameters in our multivariate sensitivity analysis, we used a uniform distribution between the high and low QALY scenarios for each decrement detailed above. For all other parameters, we used Beta-pert distributions over the ranges indicated in Table 1. We conducted simulations in @ Risk for Excel version 6.2.1. To obtain the $95 \%$ confidence interval, we conducted simulations until there was a $95 \%$ chance that the mean estimate of each output parameter was within $5 \%$ of its true value.

We also projected our results forward several years in anticipation of increased herd protection for the additional serotypes in PCV13 not in PCV7 from the childhood program. We modeled results for each year in the 6-year phase-in period for herd protection described in the "Disease Parameters" subsection.
We conducted several additional one-way sensitivity analyses to look at the responsiveness of our results to changes in potentially important parameters. Since the two available sources of QALY decrements differed by an order of magnitude, we analyzed a scenario using the higher decrements. We also analyzed a scenario where PCV13 showed much more rapid waning - no waning for 5 years followed by waning to zero over 15 years. We conducted two alternative scenarios for vaccine prices. In the first, we used the reported private price of vaccines ( $\$ 128.16$ for PCV13 and $\$ 63.47$ for PPSV23). ${ }^{24}$ In the second, we used the maximum allowable reimbursement under Medicare Part B (\$145.11 for PCV13 and \$72.35 for PPSV23). ${ }^{30}$ We also modeled a scenario where PPSV 23 was as effective (45\%) against vaccine-serotype NBP as PCV13. 


\section{RESULTS}

Results for base case and multivariate sensitivity analyses for the four strategies are presented in Table 2. Point estimates from base-case runs for each endpoint are noted and $95 \%$ confidence intervals from multivariate sensitivity analysis are reported in parentheses. Adding PCV13 to the previous schedule at ages 50,60, or 65 reduced NBP and IPD cases and related deaths. Replacing PPSV23 at 65 with PCV13 reduced NBP cases and deaths, but increased IPD cases and deaths, likely due to different serotypes covered. Each strategy shows an improvement in QALYs ranging from 1183 for the strategy that added PCV13 at age 60, up to 3053 for PCV13 at age 65, with the latter being the most health improving strategy. Total costs range from $\$ 64$ million for the strategy that replaces PPSV23 at age 65 with PCV13, to $\$ 496$ million, which provides PCV13 to adults at age 50 . The strategies that incorporated PCV13 at age 65 provided the most value for money with cost-effectiveness ratios of $\$ 62,065 /$ QALY gained for adding PCV13 at age 65 and $\$ 46,396$ per QALY gained for replacing PPSV23 at age 65 with PCV13. Total counts for outcomes from these models are available online in the Electronic supplementary material.

Figure 2 shows how the two strategies that provided the most value for money compared over time as the herd protection provided by the childhood PCV13 vaccination program manifested. The cost per QALY gained of both strategies (adding PCV13 to the previous schedule and replacing PPSV23 in the previous schedule) increased with each successive year of the program as more disease was prevented due to herd protection. The cost per QALY gained of the replacement strategy is not shown after 2015, since at that point replacing PPSV23 with PCV13 actually resulted in fewer QALYs than the previous strategy. After an additional 6 years of projected herd protection impacts from the childhood immunization program, the cost per QALY gained of adding PCV13 to the previous strategy rose to $\$ 272,621$.

Table 3 presents several one-way sensitivity analyses for the strategy that added PCV13 at age 65 years. Using alternate QALY values did not substantially impact cost effectiveness. An alternative scenario for waning immunity following a dose of PCV13 as described in the Methods section also demonstrated similar results to the base case. The cost-effectiveness ratios were more sensitive to alternative prices. When we modeled vaccine cost at the reported average private price or the maximum allowable reimbursement level under Medicare, keeping all other variables constant, cost per QALY gained increased to $\$ 96,654$ and $\$ 110,284$ respectively. The Column 6 in Table 3 presents detailed results with an additional 6 years of herd protection from the childhood immunization program for the strategy that added PCV13. With an additional 6 years of herd protection from the childhood program, the number of QALYs saved fell to 990, driven largely by dramatic declines in NBP cases prevented. Model results when PPSV23 was assumed to be $45 \%$ effective against vaccine-type NBP are shown in the final column of Table 3. In this scenario, adding PCV13 was very expensive per QALY gained, even before herd protection from the childhood immunization program fully manifested with a cost per QALY gained of \$309,211.

\section{DISCUSSION}

Adding PCV13 to the previous schedule at age 65 and replacing PPSV23 with PCV13 at age 65 provided more value for money than strategies involving vaccination at earlier ages. This is driven partly by higher disease incidence for those over age 65 in our model. However, the cost-effectiveness of both strategies was quite sensitive to projected herd protection from the childhood PCV13 immunization program. While replacing PPSV23 provided slightly more value for money for a cohort of 65-year-olds in 2013, after 1 year of this strategy, adding PCV13 at age 65 was cheaper per QALY gained. The two strategies diverged further as we modeled increased herd protection. As the addition of PCV13 is projected to become much more expensive per QALY gained, the costeffectiveness of this recommendation should be re-evaluated regularly.

Our study is closely comparable with a cost-effectiveness analysis that analyzed adding PCV13 to the schedule for adults, ${ }^{32}$ but was published before the results of the CAPiTA trial were available. Similar to our results, Smith et al. found replacing PPSV23 with PCV13 provided the most QALYs per dollar spent. This prior study was conducted based on 20072008 IPD data where the proportion of IPD caused by PCV13 serotypes was $40-50 \%$, which is far higher than the $~ 30 \%$ rates used in the present study. As the present study uses later data, it captures more of the herd protection that has accrued, benefitting adults as a result of the childhood immunization program. We also projected our findings forward 6 years, when, according to our assumptions, PCV13 serotypes account for less than $4 \%$ of IPD incidence. Another important potential difference between the two studies is that the Smith et al. analysis was performed before the vaccination schedule for adults was altered to recommend PCV13 for immunocompromised adults. PCV13 is predicted to be cost-saving for this group; therefore, they may have contributed to lowering the cost per QALYs gained calculated by Smith et al. We note that our model also projected indirect replacement effects for PCV13 similar to those effects observed for PCV7. Should replacement disease prove less common for PCV13 than for PCV7, our cost per QALY calculations may be conservative, and the strategies we analyzed could be slightly cheaper. Similarly, pneumococcal incidence may be especially difficult to predict in the presence of highly variable influenza seasons. ${ }^{33}$ Our cost per QALY calculations could also be too conservative if our pneumococcal disease rate projections are too low.

With the release of the results of the CAPiTA trial, we are able to remove one piece of uncertainty 
Table 2. Health Outcomes, Costs, and Cost Ratios for Four Vaccination Scenarios Compared to Routine Use of PPSV23 in High-Risk Adults 50-64 Years and All Adults 65 Years and Older

\begin{tabular}{|c|c|c|c|c|c|c|c|c|}
\hline & \multicolumn{2}{|c|}{ Adding PCV13 at 65} & \multicolumn{2}{|c|}{ Adding PCV13 at 50} & \multicolumn{2}{|c|}{ Adding PCV13 at 60} & \multicolumn{2}{|c|}{$\begin{array}{l}\text { PCV13 replaces PPSV23 at } \\
65\end{array}$} \\
\hline & base & $95 \% \mathrm{CI}$ & base & $95 \%$ CI & base & $95 \% \mathrm{CI}$ & base & $95 \%$ CI \\
\hline \multicolumn{9}{|l|}{ Health outcomes } \\
\hline IPD cases & -226 & $(-346,-112)$ & -277 & $(-372,-197)$ & -201 & $(-258,-142)$ & 1298 & $(1098,1703)$ \\
\hline Hospitalized NBP & -4961 & $(-6652$ & -2596 & $(-3703,-1581)$ & -2161 & $(-2839,-1237)$ & -4961 & $(-5826,4049)$ \\
\hline cases & & $-2284)$ & & & & & & \\
\hline Non-hospitalized NBP & -7252 & $(-9645$ & -4431 & $(-6276,-2998)$ & -3454 & $(-4498,-2065)$ & -7252 & $(-8452,5872)$ \\
\hline cases & & $-3319)$ & & & & & & \\
\hline Deaths due to IPD & -33 & $(-50,-17)$ & -33 & $(-43,-23)$ & -26 & $(-33,-18)$ & 184 & $(156,243)$ \\
\hline Deaths due to NBP & -332 & $(-433,-145)$ & -148 & $(-200,-82)$ & -133 & $(-173,-69)$ & -332 & $(-378,262)$ \\
\hline QALYs & 3053 & $(1727,5038)$ & 1489 & $(1154,2700)$ & 1183 & $(875,2020)$ & 1383 & $(-4655,2590)$ \\
\hline Life-years & 4627 & $(2119,5891)$ & 2197 & $(1336,2847)$ & 1825 & $(1055,2257)$ & 1883 & $(-6048,2369)$ \\
\hline \multicolumn{9}{|l|}{ Costs (million \$) } \\
\hline Total cost & 189 & $(128,261)$ & 496 & $(459,528)$ & 282 & $(261,316)$ & 64 & $(34,296)$ \\
\hline Medical costs & -132 & $(-194,-60)$ & -84 & $(-123,-55)$ & -66 & $(-92,-40)$ & -88 & $(-118,144)$ \\
\hline Vaccine costs & 321 & $(320,323)$ & 581 & $(580,583)$ & 348 & $(340,371)$ & 152 & $(151,153)$ \\
\hline \multicolumn{9}{|l|}{ Cost ratios $(\$)$} \\
\hline Cost/QALY & 62,065 & $\begin{array}{l}(26,951, \\
147,828)\end{array}$ & 333,200 & $\begin{array}{l}(159,370 \\
431,171)\end{array}$ & 238,227 & $\begin{array}{l}(131,641, \\
355,424)\end{array}$ & 46,396 & $\begin{array}{l}\text { (Cost Saving, } \\
509,987 \text { ) }\end{array}$ \\
\hline Cost/Life-year & 40,949 & $\begin{array}{l}(22,495 \\
123,292)\end{array}$ & 225,892 & $\begin{array}{l}(160,451 \\
384,497)\end{array}$ & 154,489 & $\begin{array}{l}(118,108 \\
292,570)\end{array}$ & 34,076 & $\begin{array}{l}\text { (Cost Saving, } \\
391,717)\end{array}$ \\
\hline
\end{tabular}

Each modeled strategy preserved previously recommended vaccination strategies for high-risk populations (immunocompromised adults were excluded from our analysis). Previous recommendations indicated a dose of PPSV23 at diagnosis of high-risk condition. High-risk conditions include chronic heart disease, chronic lung disease, diabetes, cerebrospinal fluid leak, cochlear implants, alcoholism, chronic liver disease, or cigarette smoking. Previous recommendations also indicated a dose of PPSV23 at age 65, provided a minimum of 5 years had elapsed since previous pneumococcal vaccination. ${ }^{31}$ The strategies here apply to all adults (except the excluded immunocompromised) and add PCV13 at age 50, 60, or 65, or replace the universally recommended dose of PPSV23 at age 65 with a dose of PCV13 at age 65. The strategies "Adding PCV13 at 65" and "PCV13 replaces PPSV23 at 65" are modeled with a cohort of 65 year-olds. "Adding PCV13 at 50" is modeled with a cohort of 50-year-olds. "Adding PCV13 at 60" is modeled with a cohort of 60-year-olds

Abbreviations: IPD invasive pneumococcal disease, PCV13 13-valent pneumococcal conjugate vaccine, PPSV23 23-valent pneumococcal polysaccharide vaccine, NBP non-bacteremic pneumococcal pneumonia, QALY quality adjusted life-year

regarding the use of PCV13 in adults; namely, the vaccine's impact on NBP. However, one of the remaining principal limitations of the present study is the impact of the childhood vaccination program on disease in adults. Following the introduction of 7-valent pneumococcal conjugate vaccine, adults saw substantial declines in vaccine serotype IPD without any change in vaccination. We have anticipated similar declines for PCV13 serotype IPD. These projected declines in disease incidence make the new schedule dramatically more expensive per QALY gained after a few additional years. It will be important to monitor the indirect protection for PCV13 going forward. Since the recommendations for adults have been altered to include PCV13, discerning which part of PCV13 serotype declines are due to increased herd protection and which are due to direct protection for adults might be difficult. Our findings, however, do support the recent ACIP

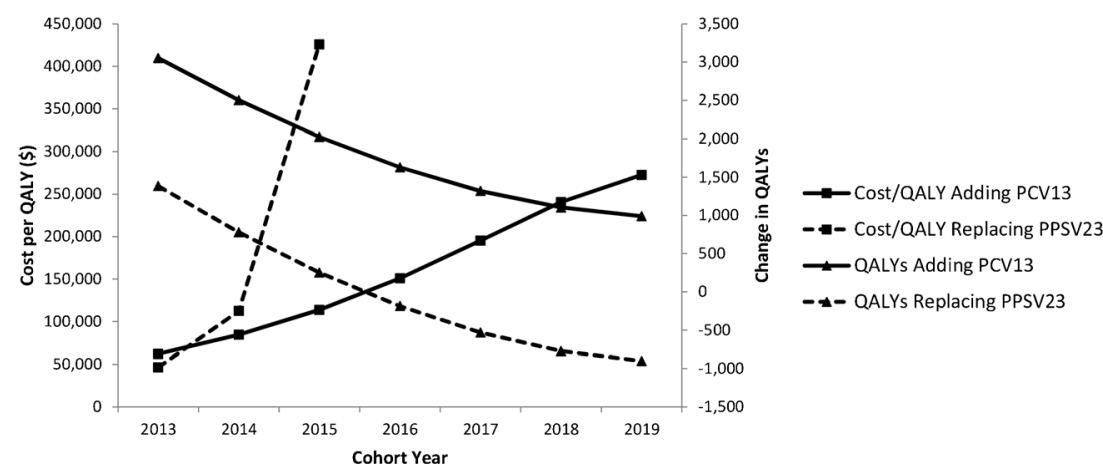

Figure 2. Cost/QALY gained and change in QALYs for adding PCV13 at age 65 or replacing PPSV23 with PCV13 at age 65 by year. Notes: Figure charts values of QALYs gained and cost-effectiveness ratios for adding PCV13 to the previous strategy or replacing PPSV23 at 65 with PCV13. Solid lines indicate the strategy that adds PCV13 at age 65. Dashed lines indicate the strategy that replaces PPSV23 at age 65 with PCV13. Triangles indicate change in QALYs and squares indicate the cost-effectiveness ratio. The values on the $x$-axis indicate the year each cohort is 65 . The principal change between years is the amount of herd protection projected from the child program. Abbreviations: PCV13 13valent pneumococcal conjugate vaccine, $P P S V 23$ 23-valent pneumococcal polysaccharide vaccine, $Q A L Y$ quality adjusted life-year. 
Table 3 Further Sensitivity Analyses-for Adding PCV13 at Age 65 Years

\begin{tabular}{|c|c|c|c|c|c|c|c|}
\hline & $\begin{array}{l}\text { Base } \\
\text { case }\end{array}$ & $\begin{array}{l}\text { Higher QALY } \\
\text { decrements }\end{array}$ & $\begin{array}{l}\text { Rapid } \\
\text { PCV } \\
\text { waning }\end{array}$ & $\begin{array}{l}\text { Vaccines cost: } \\
\text { CDC private } \\
\text { price }\end{array}$ & $\begin{array}{l}\text { Vaccines cost: } \\
\text { maximum allowed } \\
\text { by medicare }\end{array}$ & $\begin{array}{l}\text { More } \\
\text { manifested herd } \\
\text { protection }\end{array}$ & $\begin{array}{l}\text { PPSV23 } \\
\text { effectiveness vs } \\
\text { NBP }=45 \%\end{array}$ \\
\hline \multicolumn{8}{|l|}{ Health outcomes } \\
\hline $\begin{array}{l}\text { IPD cases } \\
\text { Hospitalized }\end{array}$ & $\begin{array}{l}-226 \\
-4961\end{array}$ & $\begin{array}{l}-226 \\
-4961\end{array}$ & $\begin{array}{l}-173 \\
-4687\end{array}$ & $\begin{array}{l}-226 \\
-4961\end{array}$ & $\begin{array}{l}-226 \\
-4961\end{array}$ & $\begin{array}{l}-163 \\
-1858\end{array}$ & $\begin{array}{l}-226 \\
-1547\end{array}$ \\
\hline NBP cases & & & & & & & \\
\hline $\begin{array}{l}\text { Non- } \\
\text { hospitalized NBP } \\
\text { cases }\end{array}$ & -7252 & -7252 & -6850 & -7252 & -7252 & -2715 & -2261 \\
\hline $\begin{array}{l}\text { Deaths due to } \\
\text { IPD }\end{array}$ & -33 & -33 & -25 & -33 & -33 & -24 & -33 \\
\hline $\begin{array}{l}\text { Deaths due to } \\
\text { NBP }\end{array}$ & -332 & -332 & -314 & -332 & -332 & -124 & -104 \\
\hline QALYs & 3053 & 3497 & 3014 & 3053 & 3053 & 990 & 892 \\
\hline Life-years & 4627 & 4627 & 4526 & 4627 & 4627 & 1587 & 1449 \\
\hline \multicolumn{8}{|l|}{ Costs (million \$) } \\
\hline Total cost & 189 & 189 & 198 & 295 & 337 & 270 & 276 \\
\hline Medical costs & -132 & -132 & -123 & -132 & -132 & -52 & -46 \\
\hline Vaccine costs & 321 & 321 & 321 & 427 & 469 & 321 & 321 \\
\hline \multicolumn{8}{|l|}{ Cost ratios $(\$)$} \\
\hline Cost/QALY & 62,065 & 54,183 & 65,681 & 96,645 & 110,284 & 272,621 & 309,211 \\
\hline Cost/Life-year & 40,949 & 40,949 & 43,736 & 63,764 & 72,763 & 169,974 & 190,286 \\
\hline
\end{tabular}

Each column indicates model results from a separate scenario. "Higher QALY Decrements" used higher QALY decrements for disease as detailed in the methods section. Rapid PCV Waning assumes no decline in PCV13 effectiveness for 5 years followed by waning to zero over 15 years. Vaccine cost scenarios used alternative sources for cost numbers; namely, the CDC estimate of the average private price paid for adults vaccines or the maximum reimbursement allowed by Medicare. "More Manifested Herd Protection" modeled results for a cohort of 65 year-olds in 2019 after 6 additional years of herd protection from the childhood immunization program. "PPSV23 Effectiveness vs. NBP=45\%" assumed PPSV23 has a $45 \%$ effectiveness against NBP

Abbreviations: IPD invasive pneumococcal disease, PCV13 13-valent pneumococcal conjugate vaccine, PPSV23 23-valent pneumococcal polysaccharide vaccine, NBP non-bacteremic pneumococcal pneumonia, QALY quality adjusted life-year

recommendation to add PCV13 to the schedule for all adults at the age of 65 in the short term, including the proviso that the recommendation be re-evaluated in 2018.

Acknowledgments: Dr. Stoecker's work on this project was funded by an Intergovernmental Personnel Agreement with the Centers for Disease Control and Prevention (CDC). Dr. Pilishvili, Mr. Gierke, and Dr. Kim completed the work as part of their usual duties at the CDC.

Corresponding Author: Charles Stoecker, PhD; Department of Global Health Management and PolicyTulane University School of Public Health and Tropical Medicine, 1440 Canal St \#8346, New Orleans, LA 70112, USA (e-mail: cfstoecker@tulane.edu).

\section{Compliance with Ethical Standards:}

Conflict of Interest: The authors have no conflict of interest to declare.

\section{REFERENCES}

1. National Vital Statistics Report. Deaths: Final Data for 2013. Forthcoming; 64

2. Thomas CP, Ryan M, Chapman JD, Stason WB, Tompkins CP, Suaya JA, et al. Incidence and cost of pneumonia in medicare beneficiaries. Chest. 2012;142:973-981.

3. Simonsen L, Taylor RJ, Schuck-Paim C, Lustig R, Haber M, Klugman KP. Effect of 13-valent pneumococcal conjugate vaccine on admissions to hospital 2 years after its introduction in the USA: a time series analysis. Lancet Respir Med. 2014;2:387-394.

4. Tomczyk S, Bennett NM, Stoecker C, Gierke R, Moore MR, Whitney CG, et al. Use of 13-valent pneumococcal conjugate vaccine and 23-valent pneumococcal polysaccharide vaccine among adults aged $\geq 65$ years: recommendations of the Advisory Committee on Immunization Practices (ACIP). MMWR Morb Mortal Wkly Rep. 2014;63:822-825.
5. Bonten MJM, Huijts SM, Bolkenbaas M, Webber C, Patterson S, Gault S, et al. Polysaccharide conjugate vaccine against pneumococcal pneumonia in adults. N Engl $\mathrm{J}$ Med. 2015;372:1114-1125.

6. Griffin MR, Zhu Y, Moore MR, Whitney CG, Grijalva CG. U.S. hospitalizations for pneumonia after a decade of pneumococcal vaccination. N Engl J Med. 2013;369:155-163.

7. Dirmesropian S, Wood J, MacIntyre C, Newall A. A review of economic evaluations of 13-valent Pneumococcal Conjugate Vaccine (PCV13) in adults and the elderly. Hum Vaccines Immunotherapeut. 2015;11:818825.

8. Palisade Corporation. @Risk 5.7. Newfield, NY, 2011

9. Centers for Disease Control and Prevention. Use of 13-valent pneumococcal conjugate vaccine and 23-valent pneumococcal polysaccharide vaccine for adults with immunocompromising conditions: recommendations of the Advisory Committee on Immunization Practices (ACIP). MMWR Morb Mortal Wkly Rep. 2012;61:816-819.

10. Cho B-H, Stoecker C, Link-Gelles R, Moore MR. Cost-effectiveness of administering 13-valent pneumococcal conjugate vaccine in addition to 23-valent pneumococcalpolysaccharide vaccine to adults with immunocompromising conditions. Vaccine. 2013;31:6011-6021.

11. Active Bacterial Core Surveillance (ABCs) program. Centers for Disease Control and Prevention; unpublished data, September, 2013.

12. Nelson JC, Jackson M, Yua O, Whitney CG, Bounds L, Bittner R, et al. Impact of the introduction of pneumococcal conjugate vaccine on rates of community acquired pneumonia in children and adults. Vaccine. 2008;26:4947-4954.

13. Huang SS, et al. Healthcare utilization and cost of pneumococcal disease in the United States. Vaccine 2011;29(18):3398-3412.

14. Moberley S, Holden J, Tatham DP, Andrews RM. Vaccines for preventing pneumococcal infection in adults. Cochrane Database of Systematic Reviews. 2008.

15. Huss A, Scott P, Stuck AE, Trotter C, Egger M. Efficacy of pneumococcal vaccination in adults: a meta-analysis. CMAJ. 2009; 180:48-58.

16. Williams WW, Lu P-J, O'Halloran A, Bridges CB, Pilishvili T, Hales $\mathbf{C M}$, et al. Noninfluenza vaccination coverage among adults United States, 2012. MMWR Morb Mortal Wkly Rep. 2014;63:95102. 
17. U.S. Department of Health \& Human Services. Summary Health Statistics for U.S. Adults: National Health Interview Survey, 2012. Vital and Health Statistics. 2014; 10

18. Truven Health Analytics MarketScan Database. Ann Arbor. 1994-2010.

19. Centers for Disease Control and Prevention. Prevention of pneumococcal disease among infants and children - use of 13-valent pneumococcal conjugate vaccine and 23-valent pnuemococcal polysaccharide vaccine. MMWR Morb Mortal Wkly Rep. 2010;59:1-18.

20. Fry AM, Zell ER, Schuchat A, Butler JC, Whitney CG. Comparing potential benefits of new pneumococcal vaccines with the current polysaccharide vaccine in the elderly. Vaccine. 2002;21:303-311.

21. Melagaro A, Edmunds WJ. Cost-effectiveness analysis of pneumococcal conjugate vaccination in England and wales. Vaccine. 2004;22:4203-4214.

22. Pepper PV, Owens DK. Cost-effectiveness of the pneumococcal vaccine in the United States navy and marine corps. Clin Infect Dis. 2000;30:157164.

23. Sisk JE, Riegelman RK. Cost effectiveness of vaccination against pneumococcal pneumonia: an update. Ann Intern Med. 1986;104:79-86.

24. Centers for Disease Control and Prevention. CDC Vaccine Price List. July 2013.

25. Maciosek MV, Solberg LI, Coffield AB, Edwards NM, Goodman MJ. Influenza vaccination: health impact and cost effectiveness among adults aged 50 to 64 and 65 and older. Am J Prev Med. 2006;31:72-79.

26. Bureau of Labor Statistics. Consumer Price Index - All Urban Consumers. 2012
27. Khoie T, Tiernan R, deVore N. Prevnar $13^{\mathrm{TM}}$ (PCV13): Pneumococcal 13valent Conjugate Vaccine (Diphtheria CRM197 Protein). 2011.

28. Jackson LA, Gurtman A, Cleeff M, Jansen KU, Jayawardene D, Devlin C, et al. Immunogenicity and safety of a 13-valent pneumococcal conjugate vaccine compared to a 23-valent pneumococcal polysaccharide vaccine in pneumococcal vaccine-naive adults. Vaccine. 2013;31:3577-3584.

29. Jackson LA, Gurtman A, Rice K, Pauksens K, Greenberg RN, Scott DA, Jones TR, et al. Immunogenicity and safety of a 13-valent pnuemococcal conjugate vaccine in adults 70 years of age and older previously vaccinated with 23-valent pneumococcal polysaccharide vaccine. Vaccine. 2013;31:3585-3593.

30. Centers for Medicare \& Medicaid Services. 2014 ASP Drug pricing files. 2014.

31. Bennett NM, Whitney CG, Moore M, Pilishvili T, Dooling KL. Use of 13valent pneumococcal conjugate vaccine and 23-valent pneumococcal polysaccharide vaccine for adults with immunocompromising conditions: recommendations of the Advisory Committee on Immunization Practices (ACIP). MMWR Morb Mortal Wkly Rep. 2012;61:816-819.

32. Smith KJ, Wateska AR, Nowalk MP, Raymund M, Nuorti JP, Zimmerman RK. Cost-effectiveness of adult vaccination strategies using pneumococcal conjugate vaccine compared with pneumococcal polysaccharide vaccine. JAMA. 2012;307:804-812.

33. Shrestha S, Foxman B, Weinberger DM, Steiner C, Viboud C, Rohani P. Identifying the interaction between influenza and pneumococcal pneumonia using incidence data. Sci Transl Med. 2013;5:191ra84. 